\title{
IV. Bildung und Wissenschaft
}

\author{
Ulrich Herrmann
}

\author{
„Kinderzucht“ oder „Pädagogik“? \\ Traditionelle Normierungen \\ der Erziehung und Unterweisung und \\ die innovative pädagogische Lebensalter-Konzeption \\ in der Pädagogischen Anthropologie \\ des 18. Jahrhunderts
}

Der Beginn des modernen pädagogischen Denkens im 18.Jahrhundert wird gewöhnlich mit der epochemachenden Rezeption eines Buches gleichgesetzt: mit der Rezeption von Rousseaus „Emile ou de l'éducation“, das im Jahre 1762 erschien. Die Thematik unseres Symposiums - Tradition, Norm, Innovation - legt im Hinblick auf diesen Sachverhalt genauere Rückfragen nahe: worin denn der innovative Gehalt des "Emile“ zu sehen ist, der die pādagogischen Schriftsteller in Deutschland so faszinierte; welche traditionellen Auffassungen von der Kindererziehung korrigiert bzw. aufgegeben wurden; welcher grundsātzliche Wandel in der Auffassung und Konzeptualisierung der Erziehungsbedürftigkeit und Bildungsfähigkeit der heranwachsenden jungen Menschen die bis heute anhaltende Nachwirkung Rousseaus bedingt.

Der vorliegende Beitrag soll Antworten auf diese Fragen im Rückgriff auf einige reprāsentative pădagogische Texte des ausgehenden 17. und 18.Jahrhunderts geben. Diese Texte bilden den Hintergrund für das Verständnis dafür, daß und in welchem Sinne Rousseau tatsāchlich ein paradigmatischer Wechsel des pädagogischen Denkens und Argumentierens gelungen ist.

Die rezeptionsgeschichtlich etablierte "Schwelle" - der „Emile“ -, durch die die ãltere pädagogische Tradition vor Rousseau durch dessen Wirkungsgeschichte disziplinintern abgekoppelt wurde, hat auch dazu geführt, daß die Geschichte der Anthropologie und der Psychologie im 17. und in der ersten Hälfte des 18. Jahrhunderts in Deutschland nur sehr bedingt auf die Entfaltung und Wandlung des pādagogischen
\end{abstract}


Denkens bezogen worden ist'. (Als einzige Ausnahme - noch dazu auf Frankreich bezogen - kann das Buch von Georges Snyders gelten, das in Deutschland kaum zur Kenntnis genommen und schließlich durch die oberflächlichere Darstellung von Philippe Ariès verdrängt worden ist ${ }^{2}$.) Dies hat seinen Grund vor allem auch darin, daß die - wie Dilthey sie treffend charakterisierte - „natürlichen Systeme“ einer ungeschichtlich denkenden Aufklärung mit ihren Klassifikationen der menschlichen „Kräfte“ und „Vermögen“ kaum einen Zugang finden konnten zur Erziehung und Bildung des Menschen im Horizont seiner Lebensgeschichte. Hier erfolgt der Durchbruch erst nach der Jahrhundertmitte durch $\mathrm{Herder}^{3}$ und erreicht noch vor der Wende zum 19.Jahrhundert seinen ersten Höhepunkt bei Pestalozzi in dessen Herausarbeitung der radikalen Geschichtlichkeit des Menschen, der mit dem von ihm bewirkten Wandel seiner äußeren Lebensbedingungen und -verhältnisse zugleich sich selber in seiner (inneren) Bedürfnisstruktur und in seinen Formen der Welt- und Selbstdeutung individuell und gattungsgeschichtlich „umschafft ${ }^{\star 4} . \mathrm{Daß}$ dies wiederum zusammenhängt mit der Wahrnehmung tiefgreifenden sozio-kulturellen und politisch-ökonomischen Strukturwandels (im Übergang zur „bürgerlichen Gesellschaft") sowie mit der Wahrnehmung der sich beschleunigenden Prozessualität dieses Wandels ${ }^{5}$, kann hier lediglich in Erinnerung gerufen werden und muß im folgenden immer mitbedacht werden.

\footnotetext{
${ }^{1}$ Es fehit eine unter diesem Gesichtspunkt verfaßte Geschichte der Psychologie und Anthropologie des 18. Jahrhunderts. Die ältere Literatur ist nachgewiesen bei Ulrich Herrmann, Die Rolle der Psychologie in der Entwicklung der modernen Erziehungswissenschaft (Die Psychologie des 20. Jahrhunderts, Bd. I, Zürich 1976) 1013-1026; aus der älteren Literatur immer noch heranzuziehen Max Dessoir, Geschichte der neueren deutschen Psychologie (Berlin $\left.{ }^{2} 1902\right)$; umfassende Einführungen bei Georges Gusdorf, L'avènement des sciences humaines au siècle des lumières (Paris 1973); ders., Dieu, la nature, l'homme au siècle des lumières (Paris 1972).

${ }^{2}$ Georges Snyders, Die große Wende der Pädagogik. Die Entdeckung des Kindes und die Revolution der Erziehung im 17. und 18.Jahrhundert in Frankreich (frz. orig. Paris 1965, dt. übers. Paderborn 1971); Philippe Ariès, L'enfant et la vie familiale sous l'Ancien Régime (Paris 1960, ${ }^{2} 1973$ ), dt. übers. unter dem irreführenden Titel: Geschichte der Kindheit (München/Wien 1975).

${ }^{3}$ Dokumentiert in Herders "Journal meiner Reise im Jahr 1769“" (Histor-krit. Ausgabe Stuttgart 1976).

4 In seiner Schrift „Meine Nachforschungen über den Gang der Natur in der Entwicklung des Menschengeschlechts" von 1797, jetzt leicht zugänglich in Pestalozzis „Ausgewählten Schriften“ (Stuttgart 1982). Vgl. dazu Ulrich Herrmann, Über den Gang der Geschichte in der Natur des Menschen - Einführende Überlegungen zur Geschichtlichkeit des Seelischen (Die Geschichtlichkeit des Seelischen. Der historische Zugang zum Gegenstand der Psychologie, hrsg. von Gerd Jüttemann, Weinheim 1986) 46-64.

${ }^{3}$ Dazu die Abhandlungen von Reinbart Koselleck, Vergangene Zukunft (Frankfurt ${ }^{4} 1984$ ).
} 
Daß man noch bis ins 17. und 18. Jahrhundert Kinder nicht als Kinder, sondem als „kleine Erwachsene“ wahrgenommen hätte, gehört heute zum festen Bestand historisch-pädagogischer Legendenbildung. Ein Blick auf die niederlāndische und spanische Genremalerei etwa des 16. Jahrhunderts belehrt sofort eines Besseren. Daß man Kinder und junge Leute „von Stand“ auch im 18. Jahrhundert in Kleidung und Habitus so portraitierte, als seien sie „kleine Erwachsene“, versteht sich von selbst: als Standespersonen und nicht als Kinder sollten sie gezeigt werden (auch wenn man ihnen häufig die „Insignien“ ihrer Kindlichkeit wie Spielsachen usw. beigab). Und den Sobn Gottes nicht als putto zu gestalten, verstand sich ebenfalls von selbst; denn seine Göttlichkeit mußte sich in sprechender und segnender Gebärde ausdrücken, in seiner Allmacht und Allwissenheit, und eben nicht eine in diesem Zusammenhang gānzlich absurde infantia.

Pādagogische Schriftsteller sahen schon vor 1700 Kinder als Kinder und haben manchmal mehr, manchmal weniger - Verständnis für deren kindliche Eigentümlichkeiten. Das beste Beispiel dafür ist Fénelons „Traité de l'éducation des filles“ von 1687 , in dem sich nicht nur kindgemäßer Sinn für den erziehenden Umgang mit Mädchen, sondern für Kinder ganz allgemein ausspricht, wie schon der Herausgeber der deutschen Übersetzung - kein geringerer als August Hermann Francke - in seiner Vorrede betonte ${ }^{6}$.

Die Erörterung des psychologisch-theoretischen Hintergrunds der sensualistischen tabula-rasa-Anthropologie sowie ihr Einfluß auf die Entwicklung in Deutschland muß hier wie auch unten bei John Locke weggelassen werden.

Was den Menschen „prägt“, sind die nachhaltigen „Eindrücke“ in sein Gemüt, in sein "gleichsam unbeschriebenes Gehirn“. Deshalb soll das Kind in seiner Wißbegierde angeregt werden; Unterweisung soll mit Spielen verbunden sein; durch ein "freundliches Angesicht" soll es ermuntert werden; „Vertrauen und Offenheit im Verkehr mit Kindern (ist) ersprießlicher als das schroffe Hervorkehren der Autorität"; seine Phantasie erleichtert ihm das Lernen und macht ihm Lust dazu; pedantischer Zwang wirkt abstoßend und verleidet den Kindern das Lemen; man soll den Kindern Mut machen und ihnen nicht ihr Versagen, sondern ihre kleinen Erfolge vor Augen führen, weil sie sich sonst in sich zurückziehen und energielos werden; „Belehrungen sollen ohne Strenge und Ungeduld erteilt werden“, denn ein „rauher Ton“ verdirbt das wirksamste Erziehungsmittel: ihre Empfānglichkeit für Freundschaft.

6 August Hermann Francke, Pädagogische Schriften (Paderborn $\left.{ }^{2} 1964\right)$; darin: Vorrede zu Fénelons Tractātlein von der Erziehung der Töchter (1698) $5 \mathrm{ff}$.; Kurzer und einfältiger Unterricht, wie die Kinder zur wahren Gottseligkeit und christlichen Klugheit anzuführen sind (1702) $13 \mathrm{ff}$;; Ordnung und Lehrart, wie selbige in dem Paedagogio zu Glaucha an Halle eingeführt ist (1702) $89 \mathrm{ff}$.; Instruction für die Praeceptores, was sie bei der Disciplin wohl zu beobachten haben (ca. 1713) $105 \mathrm{ff}$. Vgl. Juliane Dittrich-Jacobi, Pietismus und Pädagogik im Konstitutionsprozeß der bürgerlichen Gesellschaft. Historisch-systematische Untersuchung der Pädagogik A. H. Franckes (Diss. phil. Göttingen 1976, ms. vervielf.). 
Es ist nicht verwunderlich, daß Rousseau von Fénelon mit Hochachtung sprach; aber ist Fénelon demzufolge ein Reprăsentant ,moderner' Erziehung? Snyders hat gezeigt, daß davon keine Rede sein kann : der freundliche Umgang mit Kindern als einer cultura animi unterstellte deren menschliche Scbwäche und sollte keineswegs ihre wie es spāter heißen wird - perfectibilité als menschliche bzw. bürgerliche Vollkommenheit eröffnen. Es ging allein um die Gemütsverfassung künftiger Mütter als Erzieherinnen ihrer kleinen Kinder (woran übrigens Rousseau einleitend im „Emile“ ebenso wieder anknüpft wie die ,Ratgeberliteratur" im ausgehenden 18.Jahrhundert in Deutschland und am Beginn des 19. Jahrhunderts die systematische Erziehungslehre von F.H.C.Schwarz $)^{7}$.

Der so verstandene erziehliche und bildende Umgang mit Kindern geht aus von der Bildsamkeit ihrer "Natur" unter Berücksichtigung ihrer Neigungen und Interessen und steht deshalb vor der Frage (1) der Bewertung dieser "Natur“, (2) der Modellierung der Neigungen und Interessen (Erziehung, Zucht, „Regierung“) und (3) vor der Frage der Bestimmung des Menschen - je nach dem, wie seine Existenz eingeordnet wird: in einen göttlichen Heilsplan oder in eine säkularisiert-mundane innerweltliche Lebensführung; in eine vorgegebene Rollen- und Funktionsverteilung von Mann und Frau; in eine durch Herkommen und Stand vorgegebene Lebenssituation oder in eine fortschritts- und leistungsorientierte „bürgerliche Gesellschaft“; in eine Lebenswelt der Anpassung und Unterordnung oder in eine Lebensform der Selbständigkeit und Selbstverantwortlichkeit. Aus der Beantwortung dieser Fragen ergeben sich Erziehungsstile - generell gesprochen: Auffassungsformen vom Zusammenhang von Gott, Mensch, Natur und Gesellschaft ${ }^{B}$-, die sich nicht danach unterscheiden, wie die Kindlichkeit des Kindes und Heranwachsenden gesehen wird, sondern welchen Sinn man ihm unterlegt und welche pädagogischen Scblußfolgerungen daraus gezogen werden.

Sinn und Bedeutung bemessen sich aber daran, wie die Zukunft des Menschen antizipiert wird, und deshalb ergeben sich aus den pädagogischen Erwàgungen, wie wir sie bei Fénelon finden, zwei einander entgegengesetzte Schlußfolgerungen, zwei konträre Erziehungsstile: zum einen eine pädagogische Tradition der Ermutigung und Förderung der (geistigen) Selbstāndigkeit und Mündigkeit des Menschen und Bürgers, zum anderen eine pädagogische Tradition der Korrektur der menschlichen natürlichen „Verderbtheit“ infolge seiner Erbsündigkeit. Was der Mensch als Kind ist, zeigt sich dem pädagogischen Blick in der Perspektive dessen, was er werden soll.

Dieser Sachverhalt wird sogleich sinnenfällig, wenn zwei etwa gleichzeitig entstandene Erziehungskonzeptionen miteinander verglichen werden, die in ihrer Grundhaltung dem Kind gegenüber beide durchaus Fénelon beistimmen können.

\footnotetext{
${ }^{7}$ Friedrich Heinrich Christian Schwarz, Erziehungslehre, Bd. 1: Die Bestimmung des Menschen. In Briefen an erziehende Frauen (Leipzig 1802, ${ }^{2} 1813$ ).

8 Über den Wandel von der Anthropo-theologie zur Emanzipation des Menschen vgl. Karl Löwith, Das Verhāltnis von Gott, Mensch und Welt in der Metaphysik von Descartes und Kant (SB Heidelberger Akad. Wiss., phil.-hist. Kl., Jg. 1964, 3. Abh., Heidelberg 1964).
} 
Die eine Konzeption ist die von August Hermann Francke, dem Hallischen Theologen und Pietisten, Begründer der berühmten Hallischen Waisenhāuser und Schulen, einem der geistigen Văter eines christlich-pietistischen Erziehungsprinzips, das in weiten Kreisen des deutschen Protestantismus bis heute wirksam ist ${ }^{9}$.

Francke gibt 1698 in deutscher Übersetzung Fénelons „Traitéc heraus, aus Sorge, „wie die überall höchst verderbte Art der Kinderzucht verbessert werden könnte“. Diese Verbesserung, die auf die Verbesserung des Menschen abzielt, muß jedoch von vornherein scheitern an der Sündhaftigkeit und Verderbtheit des ,natürlichen“ Menschen. Francke schreibt in seiner Vorrede zu Fénelons Traktat:

Es ,ist nicht zu leugnen, daß alle die guten Regeln, welche der Autor gibt (i.e. Fénelon), zu nichts nutzen werden, wenn die Eltern nicht erst selbst auf ihre wahre Bekehrung und Besserung bedacht sind, ehe sie sich vornehmen, in der Erziehung ihrer Kinder etwas zu verbessern, oder wenn nicht solche Leute der Erziehung vorgesetzt werden, die gründlich zu Gott bekehrt sind. Das Werk der Erziehung ist über alle Kräfte des natürlichen Menschen. Es muß durch den Geist Gottes geführt werden; wo der im Herzen wohnt und regiert, da wird allein der rechte Grund dazu gelegt. Wenn da jemand denken wollte, daß er durch seine Sorgfalt und seinen Fleiß oder durch seine Klugheit und seinen Verstand die Kinder recht erziehen wollte, so würde es ihm am wenigsten gelingen. Es richtet es kein menschlicher Verstand aus, und auch die, die Gott fürchten, dürfen es auf ihre eigenen Kräfte nicht ankommen lassen. Das Beste muß durch das Gebet ausgerichtet werden, und derjenige steht der Auferziehung der Jugend am besten vor, der am ernstesten vor Gott hintritt und im Geist und in der Wahrheit mit Gott ringt und kämpft, daß er die Seelen, die ihm anvertraut sind, aus dem Verderben erretten möge. " 10

Auf dieser Grundlage kann Erziehung als menschliche Zwecktätigkeit gar nicht gedacht werden; denn die Voraussetzung für ihr Gelingen und ihr Ziel ist die Bekehrung zu Gott, also eine Wendung im menschlichen Leben, die gar nicht seiner eigenen Willenstätigkeit oder der seiner Erzieher, sondern einzig und allein Gottes Gnade zuzuschreiben ist.

Wie sich Gottvater dem Menschen zuwendet durch Gnade und Strafe und der Mensch sein Leben führen soll in der Furcht des Herrn, so sollen sich die Älteren der Kinder annehmen um ihrer Seele und ihrer Gottesebenbildlichkeit willen ${ }^{11}$.

Deshalb sollen Eltern und Erzieher sich den Kindern mit Liebe, Sanftmut und Geduld zuwenden; sich auch von ,ihrer Seiten in acht ... nehmen, daß sie nicht aus hitzigen und bösen Affecten über ihre Kinder und Untergebenen herfahren, denn solches ist ein Eifer mit Unverstand, und mehr eine Tyrannei als eine christliche Kinderzucht zu nennen" ${ }^{\text {"12. }}$.

${ }^{9}$ Im 19. Jahrhundert bei Cbristian Palmer, Evangelische Pädagogik (Stuttgart ${ }^{2} 1855$ ); über Kontrolle als universelles Prinzip der Gewissensbildung vgl. auch Materialien aus Schulbüchern bei Ulrich Herrmann, Kunde fürs Volk: Die Botschaft der Fibel (Volkskultur in der Moderne. Festschrift Hermann Bausinger, Reinbek 1986) 69-87.

${ }^{10}$ Francke $8 \mathrm{f}$.

11 Ebd. 14.

12 Ebd. 39. 
Kinder sollen durch freundliche Ermahnung und nicht durch Schärfe regiert werden, nach Christi Vorbild mit Worten und nicht durch Schläge. Muß aber einmal zur körperlichen Züchtigung gegriffen werden, so ist es „vonnöten, daß die Bestrafung aus einem solchen herzlichen Mitleiden herfließe, daß auch die Kinder unschwer erkennen mögen, daß man nicht seine Lust daran habe, sie zu schlagen, sondern daß man lieber alle Ruten wegwerfen und sie nur allein mit Worten ziehen wollte ${ }^{13}$.

Warum aber muß überhaupt zu solchen $Z$ wangsmitteln gegriffen werden? Weil der Zweck der Erziehung, die Hinführung der Kinder „zur wahren Gottseligkeit und christlichen Klugheit“, nur dadurch erreicht werden kann, „daß der natürliche Eigenwille gebrochen werde"14. Deshalb ist Francke auch der Auffassung, daß in seinen Waisenhäusern und Pensionaten nicht „ungeziemende Strenge“ herrsche, wie man ihm vorgeworfen hatte, „sondern vielmehr eine christliche und höchst nötige [!] Sorg-

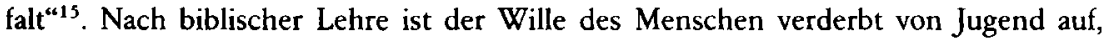
deshalb muß er gebrochen und unter Gottes Willen gebeugt werden. Erzieher und Eltern haben daher die Aufgabe, auf seine frühesten Regungen und Äußerungen zu achten. Diese müssen protokolliert und reguliert werden, was voraussetzt, daß die Kinder unablässig kontrolliert werden müssen. Disziplinierung und Kontrolle sind die universellen Prinzipien dieser Erziehungslehre, die den Menschen gehorsam und fleißig machen will, indem sie seinen Eigenwillen bricht und das ,greuliche Laster“ des Müßiggangs gar nicht erst aufkommen läßt ${ }^{16}$. Die Kinder müssen von früh bis spät in ununterbrochener nützlicher Tātigkeit gehalten werden; selbst wenn sie ausruhen und sich erholen, muß es so geschehen, daß sie „die Zeit damit nicht unnützlich vertreiben, noch ihre ohnedem flatterhaften Sinne in alle Welt zerstreuen ${ }^{\text {¿17 }}$.

Der Kern aller Erziehung ist Zucht und Disziplin „und von Gott in seinem Wort auch ernstlich anbefohlen"18. Das Ziel der Erziehung ist der fleißige, der Obrigkeit untertänige Mensch, dessen Lebensmaxime Gottvertrauen ist und nicht eitle „Weltklugheit“: diese „dreht alles nach ihrem Gutdünken“, „setzt alles auf die Spitze des Verstandes und findet doch niemals darin Ruhe, hat immer viel Streit und Zank“, und „sie ist mit ihr selbst nicht zufrieden ... und wird dadurch immer zweifelhafter "19.

Sicherlich ist zu beachten, daß Francke zunächst einmal die verwahrlosten Waisenkinder vor Augen hatte und die Aufgabe, in seinen Anstalten und Schulen auf die unabdingbare āußere Disziplin zu drāngen. Aber diese Disziplinierung war doch nur das erziehliche Medium der inneren Disziplinierung des Menschen, und insofern der „Kurze und einfältige Unterricht“ generell auch für häusliche Erziehung und Unterweisung gelten soll, ist Franckes Erziehungslehre ein Szenario von Konflikten, Zu-

${ }^{13}$ Ebd. 40.

14 Ebd. 15, 18.

${ }^{15}$ Ebd. 97.

${ }^{16} \mathrm{Vgl}$. für den größeren geschichtlichen Zusammenhang Wolfgang Dreßen, Die pädagogische Maschine. Zur Geschichte des industrialisierten Bewußtseins in Preußen/Deutschland (Frankfurt/Berlin/Wien 1982); Quellen bei Paul Münch, Ordnung, Fleiß und Sparsamkeit. Texte und Dokumente zur Entstehung der „bürgerlichen Tugenden“ (München 1984).

${ }^{17}$ Francke 32.

${ }^{18}$ Ebd. 107.

19 Ebd. 49. 
sammenstößen und Katastrophen: „mit väterlicher Zucht und liebreicher Sorgfalt über die Seelen der Kinder (zu) wachen“; es mit Worten und „Verheißungen“ zu versuchen, als ultima ratio aber immer die Rute ihr Werk tun zu lassen (und zwar so, daß die Kinder hinterher „die Hand geben, ... Dank sagen [!] und Besserung angeloben “20, damit dem heranwachsenden Mensch seine Selbstsucht und Eigensinnigkeit ausgetrieben werden, damit er seine Nichtswürdigkeit und Verderbtheit als "natürlicher Mensch" empfinden lernt und sich in der Bekehrung selber "fallen läßt". Franckes $A n$ thropo-theologie geht davon aus, daß der Mensch böse ist. Nicht der "Weltklugheit“ bedarf er, die ihn nur immer mehr verwirrt, sondern der Preisgabe des eigenen Willens. Einen eigenen Sinn in der kindlichen Eigentümlichkeit kann Francke nicht entdekken, im Gegenteil: die spezifisch kindlichen Lebensāußerungen - Spielen, Phantasieren, Trödeln usw. - sind zu bekämpfen. Wenn Francke von Liebe, Lindigkeit und Sanftmut spricht, dann meint er dies in einem christlichen Sinn - die Kinder nämlich als Geschöpfe Gottes zu achten, die eine besondere Verheißung haben -, jedoch nicht in einem pädagogiscb-psychologischen Sinn einer affektiv engen Bindung zwischen Eltern und Kindern.

Genau dies aber - eine intensivierte emotionale Bindung zwischen den Eheleuten sowie zwischen Eltern und Kindern - stellt in der Geschichte der (adligen und der bürgerlichen) Familie jene für die Pādagogik bedeutsame Entwicklung im 17. und 18. Jahrhundert dar, die für das pädagogische Denken ausschlaggebende Veränderungen und Konsequenzen mit sich brachte.

Das erste klassische Dokument dieses Verānderungs- und Umdenkungsprozesses sind die 1693 erschienenen "Some Thoughts Concerning Education“ von John Locke $^{21}$.

Hervorgegangen ist das Buch aus Briefen an den mit Locke befreundeten Gutsbesitzer Edward Clarke, der wegen der Erziehung und Ausbildung seines Sohnes um Rat gefragt hatte. Locke schrieb diese Briefe in seinem mittleren Lebensalter als erfahrener, weltlāufig gebildeter und hoch angesehener Angehöriger der britischen bürgerlichen Oberschicht, ein Politiker, Verwaltungsbeamter und Gelehrter von Rang, ein Staatstheoretiker und Philosoph von europäischem Ruf.

Der junge Clarke sollte ein gentleman werden, wie jene, die Locke selber auf ihren Bildungsreisen nach Paris und Südfrankreich begleitet hatte. In dieser dem Diesseits zugewandten Lebensform machen Überlegungen, wie Francke sie ausführte, keinen Sinn; der Rationalismus der Aufklärung lehnt die Lehre von der Erbsünde ebenso ab wie das Erziehungsziel der Bekehrung zu Gott. Die Bildung eines gentleman bezweckt ganz andere Dinge; denn von ihr hāngt „die Wohlfahrt und das Gedeihen der Nation“ $\mathrm{ab}^{22}$. Deshalb ist die Erziehung und Bildung der Jugend überall auf „die Art und Weise zu fördern ... - und zwar in stetem Hinblick auf ihre verschiedenen Lebensum-

20 Ebd. 80.

${ }^{21}$ Deutsch übers. u.d. T. Handbuch der Erziehung in: J. H. Campe, Allgemeine Revision des gesamten Schul- und Erziehungswesens (Teil 9, Wien/Wolfenbüttel 1787, Reprint Vaduz 1979), hieraus zitiert mit Angabe der $\S \S$, nicht der Seitenzahlen!

${ }^{22}$ Der Widmungsbrief an Clarke ist abgedruckt in der Ausgabe „Einige Gedanken über die Erziehung“ (Paderborn 1967) 5-7. 
stānde und Veranlagungen -, welche die leichteste, kürzeste und aussichtsreichste ist, tugendhafte, nützliche und tüchtige Männer in verschiedenen Berufen hervorzubringen. Am meisten jedoch sollte man sich um die Berufserziehung des Gentleman sorgen. Denn wenn die Angehörigen dieses Standes durch ihre Erziehung einmal auf die rechte Bahn gebracht worden sind, so werden sie bald alles übrige in Ordnungen bringen." 23

Erziehung ist Gewöhnung durch Übung (38, 45, 66 u. ö.), deren Ziel es ist, der Natur und der Vernunft gehorchen zu lernen ( $31 \mathrm{ff}$.), so daß der Mensch eines Tages selbstāndig und mündig ist, sich selber überlassen werden kann (42). Diese übende Gewöhnung geschieht mit Freundlichkeit und Festigkeit, durch Belohnungen und Strafen, Lob und Tadel, Vorbild und Beispiel. Zuviel Strenge ist pädagogisch sinnlos und in der Wirkung negativ (46): ,wenn die Gemüther der Kinder zu sehr gebeugt und gedemüthigt werden, wenn all ihr Muth durch ein Übermaaß von Strenge niedergeschlagen wird, so erschlafft alle ihre Spannkraft und Thätigkeit“. „... sklavische Zucht macht sklavische Gemüther" (50), „Schläge also und alle andre Arten von sklavischen und körperlichen Strafen sind keine schickliche Zucht, wenn es darum zu thun ist, weise, gute und edeldenkende Menschen zu erziehen“ (52).

Kinder sind Kinder: ihr Spielen, ihr fröhliches Wesen und ihre Natürlichkeit [!] sollen gefördert werden; man soll ihnen nichts andressieren oder „aufpflastern“ oder an Fehlern herumlaborieren, die die Zeit von selber heilen wird (67). Sie lernen durch Lust und Interesse, nicht durch Last und Leistungsdruck (73).

Aber Kinder sind nicht nur Kinder, sondern sie wollen erwachsen werden und beizeiten als Erwachsene behandelt werden. Locke weist verschiedentlich auf dieses besondere Beziehungsproblem zwischen Eltern und Kindern hin: daß die Kinder nämlich gleichberechtigt sein wollen; daß man sie als vernünftige Personen mit der ihnen eigentümlichen Klugheit und Menschenkenntnis achten muß, wenn man ihre Achtung genießen will (71); „daß Kinder allemal eher reif seyn wollen, als man glaubt“ (ebd.), und sie wünschen „früher als man glaubt, wie vernünftige Geschöpfe behandelt zu seyn" (81). Und von daher kommt Locke zu ersten Hinweisen auf eine lebensalterspezifische Erziehung ( $35 \mathrm{f}$ ).

Bildung des Geistes und Charakters steht im Mittelpunkt von Lockes Pädagogik; sie zielt auf Tugend, Weisheit und gute Lebensart im Rahmen eines angenehmen und für die Öffentlichkeit nützlichen Lebens. Nicht die Abtötung der Natürlichkeit und die Bekehrung wie bei Francke stehen im Zentrum, sondern - ganz im Gegenteil - der Erwerb von „Weltklugheit" und Weltläufigkeit; die Religion rückt in diesem Erziehungsplan ganz an den Rand. An die Stelle der Antbropo-theologie (Löwith) tritt das Interesse an aufgeklärter Mündigkeit, das pädagogische Interesse wendet sich der Individualität des Menschen zu und erstrebt seine intellektuelle und tugendhafte Selbsttätigkeit und Selbständigkeit. Aus diesem Selbstbewußtsein des sich emanzipierenden Bürgertums wird Locke der Begründer einer bürgerlich-liberalen Erziehungs- und Bildungstradition, die in Deutschland im 18. Jahrhundert neben der durch Francke begründeten Tradition rezipiert wurde; denn so sehr man die christlich-pietistische

${ }^{23}$ Ebd. $6 \mathrm{f}$. 
Erziehungslehre und „Kinderzucht" trefflich einsetzen konnte, um die Menschen zu verfleißigen, so wenig entsprach sie doch der eigenen sozialen, ökonomischen und mentalitären Lage des Bürgertums. Hier kann Locke mit seinen Überlegungen normund traditionsbildend wirken:

Mit der Kritik an der Schule, die entweder nichts taugt oder nichts Nutzbringendes vermittelt und demzufolge Zeitverschwendung ist; mit seinem Plädoyer für nützliche Kenntnisse einschließlich kaufmännisches Rechnen und Buchhaltung, für die modernen Fremdsprachen, die der Handel- und Gewerbetreibende nun einmal benötigt ebenso wie geographische, historische und juristische Kenntnisse usw. Und dies alles auf den individuellen Fall zugeschnitten (216), den nun jeder Vater für jeden seiner Söhne sorgfältig planen und überlegt ausführen muß.

Der soziale Status und Rang der bürgerlichen Familie, besonders bei den vermögenslosen Beamten, Gelehrten und Akademikern, kann nur durch eine qualitătvolle Bildung und Ausbildung der Kinder erhalten werden. Deren Lebensweg muß sorgfältig geplant werden: bei den Söhnen die berufliche Ausbildung und die Wahl der richtigen "Lebensart" (Beruf), bei den Töchtern die Vorbereitung auf ihre dreifache Rolle als Gattin, Hausfrau und Mutter durch die Bildung des Herzens. Die Devise der bürgerlichen Erziehung und Bildung im 18.Jahrhundert lautet: „Die Zukunft vorbereiten! ${ }^{\text {[24 }}$

Diderot lāßt seinen „Père de famille“ dem Sohn sagen: „Ich habe dich nicht einer käuflichen (Amme) übergeben; ich selbst habe dich sprechen und denken gelehrt; in dem Maße, wie du älter wurdest, habe ich deine Neigungen beobachtet und auf sie den Plan deiner Erziehung aufgebaut; ich habe ihn rastlos verfolgt. Wie viele Sorgen habe ich mir gemacht, um sie dir zu ersparen. “25

Im Artikel „Père“ in der „Encyclopédie“ heißt es: „Vāter, die reich geworden sind oder die den Aufstieg ihrer Familie betreiben, lieben ihre Kinder zārtlicher, zweifellos weil sie sie unter zwei gleich interessanten Gesichtspunkten ansehen: als ihre Erben und als ihre Geschöpfe."

Die bürgerliche Lebenswelt setzt Kräfte frei, die die überkommenen Lebensformen tiefgreifend verändern: die Intimisierung der innerfamilialen Beziehungen und die elterliche Sorge und Liebe für ihre Kinder ${ }^{26}$ (oder richtiger: für deren Zukunft und damit für die eigene Zukunft).

Einer der einflußreichsten pädagogischen Schriftsteller im deutschsprachigen Bereich, Johann Georg Sulzer, erinnert sich in seinen Lebenserinnerungen an den bil-

${ }^{24}$ Snyders 250.

${ }^{25}$ Nachweise für das Folgende, auch der Zitate, bei Ulrich Herrmann, Über „bürgerliche Erziehung " in Frankreich und Deutschland im 18. Jahrhundert (Aufklärungen. Frankreich und Deutschland im 18. Jahrhundert, Bd. 1, Heidelberg 1986) 47-63; ders., „Die Bildung des Bürgers“. Die Formierung der bürgerlichen Gesellschaft und die Gebildeten im 18. Jahrhundert (Weinheim/Basel 1982); Wolfgang Ruppert, Bürgerlicher Wandel. Studien zur Herausbildung einer nationalen deutschen Kultur im 18. Jahrhundert (Frankfurt/New York 1981).

${ }^{26}$ Edward Shorter, Die Geburt der modernen Familie (New York 1975, dt. übers. Reinbek 1977); Elisabeth Badinter, Die Mutterliebe. Geschichte eines Gefühls vom 17. Jahrhundert bis heute (Paris 1980, dt. übers. München 1981). 
denden Einfluß, den sein Vater auf seine große Familie nahm, wenn er sie um sich versammelte; ebenso erinnert er sich an die Sanftmut und Liebe der Mutter: „So oft ich auch als ein kleines Kind Abschied von ihr nahm, um in die Schule, oder zu meinen Kameraden zu jugendlichen Spielen zu gehen, erinnerte mich die rechtschaffene Frau auf's zärtlichste, mich wohl in Acht zu nehmen, nichts zu reden oder zu thun, dessen ich mich hernach würde zu schämen haben. “"

Sulzer beschreibt die Verhältnisse in seinem bürgerlichen Elternhaus in Winterthur in den 1720 er Jahren, und sie sind gewiß vorbildlich gewesen für seine eigene Hauslehrerpraxis. Sein 1745 erstmals erschienener „Versuch von der Erziehung und Unterweisung der Kinder“28, der auf Lockes „vortreffliches“ Buch zurückgreift, formuliert jedenfalls die Erziehungs- und Bildungsgrundsätze für Kinder als (künftige) Bürger und Menschen. Die Regeln und Ziele stimmen im wesentlichen mit denen Lockes überein, differenzierter ist seine Betrachtung der Lebensalter, die zum ersten $\mathrm{Mal}$ in der neueren pädagogischen Literatur einer eingehenderen psychologischen Deutung in pädagogischer Absicht unterzogen werden ${ }^{29}$ :

Drei Stufen der „Kindheit“: (1) vom 1.-3. Lebensjahr: eine Zeit, wo die Kinder ihren Eigensinn ablegen müssen (auch durch Zwang und Strafen), gehorsam werden sollen und ihre Eltern lieben lernen; das Hauptmittel der Erziehung ist die Liebe und Zuneigung zu den Kindern, ihren Gehorsam bestärkt man durch „Liebesbezeugungen“; (2) 4.-6. Jahr: die Zeit, in der Kinder Ordnung, Fleiß, Genauigkeit lernen sollen und wo sie selber unter Kindern leben wollen; (3) 6.-10. Jahr: Jahre, wo man ihren Leib abhärten und üben soll; wo man vernünftig mit ihnen sprechen kann, wo sie selber die ersten Gründe der Moral und der Tugend einsehen lernen können. "Je älter die Kinder werden, je weniger mechanisch muß man mit ihnen umgehen.“ (S.152)

Zwei Stufen des Jugendalters: (1) 11.-14. Jahr, ein Alter, in dem die Heranwachsenden schon deutlicher zeigen, „was für Leute sie werden wollen“ (S. 154); sie sind schon halb erwachsen und vernünftig, jetzt muß man sie sich selber überlassen, damit sie „lernen, sich selbst zu regieren" (ebd.); sie sollen jetzt in „mäßiger Freiheit" (ebd.) leben; (2): letzte Phase, in der ihre Urteilsfähigkeit und ihr Geschmack sich so formen sollen, daß sie erwachsen sind.

Sulzer entwickelt seine Erziehungs- und Unterrichtslehre als eine differenzierte pädagogisch-psychologische Analyse der Lernformen und -inhalte für die verschiedenen Zeitabschnitte des Kindes- und Jugendalters - sozusagen chronologisch nach der Ordnung der Natur und der Vernunft gemäß deren Eigengesetzlichkeit. Wenig später führt Johann Friedrich May in seinem zweibändigen Werk „Die Kunst der vernünftigen Kinderzucht“30 - soweit sich überblicken läßt - zum ersten Mal in der pädagogischen Literatur des 18. Jahrhunderts in Deutschland eine antbropo- bzw. psycho-genetische Betrachtungsweise ein.

${ }^{27}$ Jobann Georg Sulzers Lebensbeschreibung, von ihm selbst aufgesetzt (Berlin/Stettin 1809) 10.

${ }^{28}$ Hier zitiert nach dem Wiederabdruck in Sulzers Pädagogischen Schriften (Langensalza 1922).

${ }^{29}$ Ebd. $125 \mathrm{ff}$. im 8. Kapitel über die „verschiedenen Stufen des Alters“.

30 Jobann Friedrich May, Die Kunst der vernünftigen Kinderzucht in den nöthigsten Grundsätzen abgefaßst (2 Theile, Helmstedt 1753/54). 
Die Natur bringt ein lebendiges Wesen nicht mit einem Mal hervor, sondern indem sie es "nach und nach in Ansehung der Theile und Kräfte so zu reden auswickelt (sic!)“. „Dahero man ihre Wirkungen nach der Zeit (sic!) abmessen kann“ (§ 41).

Die „Wirkungen nach der Zeit“ verweisen auf den biographischen Lebenszusammenhang des Menschen. Deshalb ist für das pädagogische Sehen und Denken nicht mehr nur die Klassifikation der seelischen und geistigen Kräfte und Vermögen ausreichend, sondern unabdingbar ist für den Erzieher, daß er bewandert sein muß in der "Charakteristick“ der Menschen (§ 47). Diese aber läßt sich nur verstehen, wenn man zu den Unterschieden im „Naturell“ die Unterschiede des Alters, des Standes und vor allem der materiellen Lebensumstānde (arm/reich) hinzunimmt („Von dem Besonderen in der Erziehung“, S.110ff.).

Daraus ergeben sich dann die pädagogisch sinnvollen Umgangsformen mit Kindern und Heranwachsenden in der Tradition von John Locke.

Dieses genetische Prinzip hat sich dann gegen Ende des 18. Jahrhunderts in der anthropologischen Literatur weitgehend durchgesetzt ${ }^{31}$.

Der Blick auf die Lebensgeschichte des Menschen als sein Lebensweg in eine offene Zukunft - und umgekehrt: das Verstāndnis der Zukunft als eines geschichtlich-gesellschaftlich offenen Horizontes der Gestaltung von Leben und Gesellschaft - setzt die Sorge der (bürgerlichen) Eltern für ihre Kinder frei und verbindet jene mit diesen in liebevoller Zuwendung. Diese Sorge und Liebe haben nur dann einen Sinn, wenn man die Kinder nicht länger in ihrer Schwāche (und Verderbtheit) betrachtet (wie Francke) oder - ebenfalls neutestamentlich gesehen - in ihrer Reinheit und Unschuld (wie Fénelon ${ }^{32}$ ), sondern indem man ihnen vertraut ${ }^{33}$. Erst das Vertrauen darauf, daß der Mensch sich vervollkommnen kann, daß er dadurch seinen Lebensweg zu meistern weiß und demzufolge dann auch die gesellschaftlichen Verhältnisse zu seiner Zufriedenheit gestalten und in Ordnung halten kann (Locke), verleiht der Vorbereitung auf das Erwachsensein seine besondere Bedeutung, und erst in dieser Perspektive versteht man den Wechsel von der „Kinderzucht“ zur „Pädagogik“: einer Pädagogik der Entwicklung und Förderung, Bildung und Emanzipierung eines selbständigen und selbsttätigen bürgerlichen Subjekts und seiner intellektuellen und kulturellen Individualitāt. In dieser Perspektive wird auch das Pathos des Fortschritts, das besonders in der deutschen Spātaufklärung bei Kant geschichtsphilosophisch und gesellschaftstheoretisch mit der Pädagogik einherging (und bis heute vielfach einhergeht), verständlich; „denn hinter der Edukation steckt das große Geheimnis der Vollkommenheit der menschlichen Natur ... Es ist entzückend, sich vorzustellen, daß die menschliche Natur immer besser durch Erziehung werde entwickelt werden, und daß man

\footnotetext{
${ }^{31}$ Statt vieler: Jobann Nicolas Tetens, Philosophische Versuche über die menschliche Natur und ihre Entwickelung (Leipzig 1777); Peter Villaume, Geschichte des Menschen (Dessau/Leipzig 1783); J. Ith, Versuch einer Anthropologie oder Philosophie des Menschen nach seinen körperlichen Anlagen (2 Theile, Bern 1794/95); weitere Nachweise bei Herrmann (1976, wie Anm. 1).

${ }^{32}$ Robert Spaemann, Reflexion und Spontaneitāt. Studien über Fénelon (Stuttgart 1963) 138 ff.: Der „Geist der Kindheit" und die Entdeckung des Kindes.

${ }^{33}$ Snyders $250 \mathrm{ff}$.
} 
diese in eine Form bringen kann, die der Menschheit angemessen ist. Dies eröffnet uns den Prospekt zu einem künftigen glücklichern Menschengeschlechte.“"34

Die Frage, ob der Mensch von „Natur" aus gut oder böse sei, ist hinfällig geworden: auf „Natur" lassen sich Kategorien der Moral nicht anwenden. Die Antbropo-theologie hat abgedankt; es bleibt die Frage, was der Mensch - individuell und gattungsgeschichtlich - werden soll und wie er es werden kann und was ihn daran hindert, es zu werden.

\section{III}

Wenn dies der geschichtliche Gang des sich aufklärenden (bürgerlich-)pädagogischen Denkens von der „Kinderzucht“ zur „Pädagogik“ im 18.Jahrhundert war - der Gedanke einer selbstreflexiven „Bildung“ und seiner anders gelagerten und begründeten anthropologisch-kategorialen Fassung sei einmal ausgeklammert, da er in andere geistesgeschichtliche Zusammenhänge führen würde -, dann bleibt die Antwort auf die Frage zu geben, worin denn die paradigmatische Wendung des pädagogischen Denkens bei Rousseau zu sehen ist, wie sie auch von den Zeitgenossen - besonders Kant! - wahrgenommen wurde.

Das Neue bei Rousseau war offensichtlich nicht - wie unser Blick in die Literatur des 17. und 18. Jahrhunderts gezeigt hat - die Wahrnehmung des Kindes als Kind und auch nicht die genetische Psychologie der Lebensalter. Neu und faszinierend war - und hierin epochemachend - die Konstruktion einer fiktiven Biographie sub specie educationis: die Einbettung des erziehenden Umgangs und des Lemens in eine sorgfältig gestaltete „Lernumwelt"; die Einbettung der "natürlichen“ Entwicklung in ein pädagogisch reflektiertes Arrangement der Weltaneignung und Selbsterfahrung; die Einbettung des pädagogischen Reflektierens und Handelns in Abwägungen und Antizipationen des Zusammenhangs von Intentionen und möglichen Wirkungen im künftigen Leben des Educandus.

Damit nimmt Rousseau einen entscheidenden Perspektivenwechsel vor: Bis dahin war die Erziehungslehre eine Sammlung von Regeln und Rezepten, die, wenn man sie nur richtig in Anwendung brachte, unfehlbar ein bestimmtes Ergebnis zeitigen mußte. Erziehung als Handeln am Menschen wird verstanden als zwischenmenschliche Praxis, die ein Subjekt - den erfahrenen Erwachsenen - und ein ,Objekt ${ }^{t}$ - das unerfahrene Kind - hat. Das Handeln des Erwachsenen ist Ursache für Wirkungen, die als Verhaltensweisen des Kindes und Heranwachsenden ablesbar sind. Daß dies sich tatsäcblich so verhält, ist ganz unwahrscheinlich: nicht nur weil das Geflecht von Erfahrungen, Einflüssen und intentionalen Einwirkungen sehr viel komplexer ist und weil deren intrapersonale Verarbeitung und ihr Ergebnis als individueller Habitus nicht nach einem einfachen kausalen Ursache-Wirkungs-Schema erklärt werden kann, sondern weil das ,Objekt' der Erziehung eben ein Subjekt ist, das auf das Handeln und Denken der Erwachsenen selber aktiven Einfluß nimmt. Der Wechsel der Betrach-

34 Immanuel Kant, Über Pādagogik (Werke in 6 Bānden, Bd. VI, Darmstadt 1964) 700. 
tungsperspektive besteht mithin darin, Erziehung und Bildung aus der Sicht des Heranwachsenden zu analysieren, so daß die Erziehungslehre nicht länger eine Sammlung von Regeln und Rezepten sein kann, sondern in eine genetische Anthropologie als Entwurf eines Lebenslaufs verwandelt werden muß. Dieser Perspektivenwechsel wird zutreffend mit der Formel einer „Pädagogik vom Kinde aus"bezeichnet. Die neue Erziehungslehre soll Eltern und Erzieher pädagogisches Denken lehren, damit sie sich auf die unbekannte, sich erst entwickelnde Individualität ihrer Kinder einstellen lernen, und sie nimmt den Eltern und Erziehern die Gewißheit auf die Richtigkeit ihres Handelns.

Damit nimmt Rousseau einen weiteren entscheidenden Perspektivenwechsel vor: die erste Erziehung soll „purement négative“ $\operatorname{sein}^{35}$; sie soll Zeit verlieren und nicht gewinnen; sie soll „die Kindheit im Kinde reifen“ lassen; menschliches Handeln soll nicht in "dem Werk der Natur" herumpfuschen: „Tout est bien, sortant des mains de l'auteur des choses: tout dégénère entre les mains de l'homme." ${ }^{36}$ Und damit sind die Hinweise gegeben auf den konzeptionellen Hintergrund in Rousseaus Denken: Da er dem Gedanken des Fortschritts in Kultur und Gesellschaft skeptisch gegenübersteht ${ }^{37}$ und die Menschlichkeit des Menschen, die er als „homme naturel" hatte und die im Zustand der Vergesellschaftung defekt ist ${ }^{38}$, unter der Perfektionierung der bürgerlichen Brauchbarkeit fortgesetzt Schaden nimmt, kann der Sinn der Erziehung nicht darin bestehen, den jungen Menschen möglichst rasch auf die bürgerliche Existenz in einer ungewissen Zukunft vorzubereiten. Das könnten Erzieher und Eltern gar nicht verantworten, sie würden „barbarisch“ handeln ${ }^{39}$. Die Erziehung soll vielmehr den $e i$ genen Sinn jeder einzelnen Lebensstufe beachten und das Kind bzw. den Heranwachsenden diese Zentriertheit jeder Lebensstufe als einer in sich ruhenden Lebensform erleben und ausleben lassen - ausleben aber auch in dem anderen Sinne des Wortes, daß man sie ablegt: ein kindischer Erwachsener ist lācherlich, so wie umgekehrt ein pedantisches oder ein frühreifes Kind als ,unnormal' empfunden wird.

Rousseau hat nicht die Kinder als Kinder "entdeckt“, sondern den anthropologisch-pädagogischen Sinn der Kindbeit und des Jugendalters ${ }^{40}$; im "Vorwort" zum "Emile“ sagt er ja nicht, man kenne „die Kinder“ nicht, sondern: „Man kennt die Kindbeit (enfance) nicht. " ${ }^{\text {"11 }}$ Und so erschließt Rousseau auch den Sinn des (bürgerlichen) Jugendalters: die Pubertāt als Reifezeit, in der die in Erotik verwandelte Sexualität als Beherrschung der Leidenschaften die Voraussetzung ist für ein glückliches Le-

${ }^{35}$ Jean-Jacques Rousseau, Emile ou de l'éducation (1762, jetzt in den Oeuvres complètes [Pléiade], Bd. IV) 323; dt. übers. Emil oder über die Erziehung (Paderborn 1971) 72.

${ }^{36}$ Anfangssatz im 1. Buch des „Emile“.

${ }^{37}$ Im 1. Discours von 1750: Hat der Wiederaufstieg der Wissenschaften und Künste zur Läuterung der Sitten beigetragen?

${ }^{38}$ Dazu die Aufsätze von Robert Spaemann in seiner Sammlung: Rousseau - Bürger ohne Vaterland (München 1980).

39. Emile (dt. übers.) 55.

40 Ulrich Herrmann, Kindheit und Jugend im Werk J. H. Campes. Pādagogische Anthropologie und die „Entdeckung“ des Kindes im Zeitalter der Aufklärung (Neue Sammlung 15, 1975) 464-481; wiederabgedr. in ders. (1982, wie Anm. 25) $178 \mathrm{ff}$.

${ }^{11}$ Emile (dt. übers.) 5. 